\title{
Manejo não-farmacológico da dor em neonatos internados em Unidades de Terapia
}

\section{Intensiva: atualidades}

\author{
Non-pharmacological pain management in neonates admitted to Intensive Care Units: update \\ Manejo no farmacológico del dolor en recién nacidos ingresados en Unidades de Cuidados \\ Intensivos: actualización
}

Recebido: 26/09/2021 | Revisado: 02/10/2021 | Aceito: 20/11/2021 | Publicado: 01/12/2021

Thyago de Oliveira Afonso

ORCID: https://orcid.org/0000-0001-7616-9011

Universidade Federal de Pernambuco, Brasil

E-mail: thyago.oafonso@gmail.com

Samuel Lopes dos Santos

ORCID: https://orcid.org/0000-0003-3375-9171

Universidade Federal do Piauí, Brasil

E-mail: samuellopes121314@gmail.com

Gustavo Baroni Araújo

ORCID: https://orcid.org/0000-0002-3162-7477

Universidade Estadual de Londrina, Brasil

E-mail: gustavobaroni13@hotmail.com

Layanne Cavalcante de Moura

ORCID: https://orcid.org/0000-0003-2781-1076

Faculdade Integral Diferencial, Brasil

E-mail: layannecavalcante@ hotmail.com

Guilherme Dantas Borges

ORCID: https://orcid.org/0000-0002-3415-8725

Universidade de Vassouras, Brasil

E-mail: gdantasborges@gmail.com

Joelma Maria dos Santos da Silva Apolinário

ORCID: https://orcid.org/0000-0001-9521-9432

Centro Universitário Maurício de Nassau, Brasil

E-mail: jo.silva00@hotmail.com

Samara Atanielly Rocha

ORCID: https://orcid.org/0000-0002-5622-9280

Faculdade de Saúde e Humanidades Ibituruna, Brasil

E-mail: samaraatanielly@ outlook.com

Ronnyele Cássia Araujo Santos

ORCID: https://orcid.org/0000-0001-9203-2680

Centro Universitário Maurício de Nassau, Brasil

E-mail: ronnyeleenfermagem@gmail.com

Caroliny Ferreira Lira

ORCID: https://orcid.org/0000-0001-7907-9791

Secretaria de Saúde do Recife, Brasil

E-mail: liracarolinyferreira@gmail.com

Ilana Marjorie Macedo Borges Miranda

ORCID: https://orcid.org/0000-0002-3576-6474

Centro Universitário UNINOVAFAPI, Brasil

E-mail: ilana_miranda10@hotmail.com

André Lucas Santos Rocha

ORCID: https://orcid.org/0000-0002-9665-728X

Centro Universitário UNINOVAFAPI, Brasil

E-mail: andre.rocha95@hotmail.com

Ricardo Pessoa Rocha Melo

ORCID: https://orcid.org/0000-0002-7868-9325

Universidade Federal do Piauí, Brasil

E-mail: ricardo.fisio5@hotmail.com

Cedric Adam Spíndola de Araújo Viana

ORCID: https://orcid.org/0000-0003-0404-1144 Centro Universitário UniFacid, Brasil

E-mail: cedricadam8@ hotmail.com

Denise Delmonde Medeiros

ORCID: https://orcid.org/0000-0003-1453-1024

Universidade Federal do Piauí, Brasil 


\author{
Winícius de Carvalho Alves \\ ORCID: https://orcid.org/0000-0003-3118-5696 \\ Universidade Federal de Pelotas, Brasil \\ E-mail: winiciusdecarvalho@ hotmail.com
}

\begin{abstract}
Resumo
Introdução: Inerente à maioria dos procedimentos realizados em Unidade de Terapia Intensiva Neonatal, a dor é um fenômeno que precisa ser correta e precocemente avaliado e tratado para que não prejudique o desenvolvimento do bebê a longo prazo. A sua terapêutica não-farmacológica age no bloqueio da transmissão do estímulo nociceptivo ao tálamo e na ativação de vias inibitórias descendentes, sendo uma importante e diversa aliada no controle da dor. Metodologia: Trata-se de uma Revisão Integrativa de Literatura feita com pesquisa inicial das bases de dados Literatura Latino Americana e do Caribe em Ciências da Saúde (LILACS), Scientific Electronic Library Online (SciELO) e Medical Literature Analysis and Retrieval Sistem (Medline). Foi utilizado o termo de busca "Intensive Care Units, Neonatal AND Pain management”. Incluiu-se publicações de 2017 a 2021 e excluiu-se artigos revisão, metanálises e relatos de casos. Não houve duplicações. Excluiu-se, ainda, os artigos cujo título ou resumo corroboravam com o propósito desta revisão. Organizou-se os 18 artigos filtrados pelos critérios de exclusão e, a leitura de todo seu conteúdo, resgatou-se 14 artigos que compõem os resultados dessa revisão. Resultados e Discussão: Todos os artigos resgatados são randomizações e a maioria delas foi publicada no ano de 2020. Observouse que a punção de calcanhar é um procedimento doloroso e avaliado na maioria dos ensaios clínicos, inclusive com aplicação da escala Premature Infant Pain Profile (PIPP), para os estudos com pré-termos. Ainda, constatou-se que sacarose oral a $24 \%$ foi a intervenção mais comparada a outras e que se mostrou inferior ou equiparável ao método canguru e a ingesta de leite materno para o controle da dor em neonatos internos em UTI. Conclusão: esta revisão pontuou a eficácia clínica de intervenções de diferentes naturezas evidenciadas nos estudos resgatados. O olfato, a audição, o paladar e o tato têm sido potenciais campos de intervenção para o controle analgésico ou distração da dor em neonatos submetidos a procedimentos dolorosos de rotina. Destaca-se que o método canguru, a ingesta de leite materno e o seu odor, bem como o som do batimento cardíaco materno têm mostrado resultados positivos, seguros e reproduzíveis, inclusive em comparação com o uso de sacarose oral a $24 \%$. Ressalta-se que novos ensaios clínicos e metanálises são imprescindíveis para o fortalecimento das conclusões desse estudo.
\end{abstract}

Palavras-chave: Unidades de Terapia Intensiva; Neonatal; Manejo da dor; Pediatria.

\begin{abstract}
Introduction: Inherent in most procedures performed in the Neonatal Intensive Care Unit, pain is a phenomenon that needs to be correctly and early evaluated and treated so that it does not harm the baby's development in the long term. Its non-pharmacological therapy does not block the transmission of nociceptive stimuli to the thalamus and activates descending inhibitory pathways, being an important and diverse ally in pain control. Methodology: This is an Integrative Literature Review carried out with an initial search of the Latin American and Caribbean Literature in Health Sciences (LILACS), Scientific Electronic Library Online (SciELO) and Medical Literature Analysis and Retrieval System databases (Medline). The search term "Intensive Care Units, Neonatal AND Pain management" was used. Publications from 2017 to 2021 were included and review articles, meta-analyses and case reports were excluded. There were no duplications. Articles whose title or abstract corroborated the purpose of this review were also excluded. The 18 articles filtered by the exclusion criteria were organized and, after reading all of their content, 14 articles that make up the results of this review were retrieved. Results and Discussion: All retrieved articles are randomizations and most of them were published in 2020. It was observed that heel puncture is a painful procedure and evaluated in most clinical trials, including the application of the Premature Infant Pain Profile scale (PIPP), for studies with preterms. Furthermore, it was found that $24 \%$ oral sucrose was the intervention most compared to others and that it was inferior or comparable to the kangaroo method and the intake of breast milk for pain control in neonates hospitalized in the ICU. Conclusion: this review pointed out the clinical effectiveness of interventions of different natures evidenced in the rescued studies. Smell, hearing, taste and touch have been potential fields of intervention for analgesic control or pain distraction in neonates undergoing routine painful procedures. It is noteworthy that the kangaroo method, the intake of breast milk and its odor, as well as the sound of the maternal heartbeat have shown positive, safe and reproducible results, even in comparison with the use of oral sucrose at $24 \%$. It is noteworthy that new clinical trials and meta-analyses are essential to strengthen the conclusions of this study.
\end{abstract}

Keywords: Intensive Care Units; Neonatal; Pain Management; Pediatrics.

\title{
Resumen
}

Introducción: Inherente a la mayoría de los procedimientos que se realizan en la Unidad de Cuidados Intensivos Neonatales, el dolor es un fenómeno que necesita ser evaluado y tratado de manera correcta y temprana para que no perjudique el desarrollo del bebé a largo plazo. Su terapia no farmacológica no bloquea la transmisión de estímulos nociceptivos al tálamo y activa vías inhibidoras descendentes, siendo un aliado importante y diverso en el control del dolor. Metodología: Revisión Integrativa de Literatura realizada con una búsqueda inicial de las bases de datos de Literatura Latinoamericana y Caribeña en Ciencias de la Salud (LILACS), Biblioteca Electrónica Científica en Línea (SciELO) y Sistema de Análisis y Recuperación de Literatura Médica (Medline). Se utilizó el término de búsqueda 
"Unidades de cuidados intensivos, manejo neonatal y del dolor". Se incluyeron publicaciones de 2017 a 2021 y se excluyeron artículos de revisión, metanálisis e informes de casos. No hubo duplicaciones. También se excluyeron los artículos cuyo título o resumen corroboró el propósito de esta revisión. Se organizaron los 18 artículos filtrados por los criterios de exclusión y, tras leer todo su contenido, se recuperaron los 14 artículos que componen los resultados de esta revisión. Resultados y discusión: Todos los artículos recuperados son aleatorizaciones y la mayoría de ellos fueron publicados en 2020. Se observó que la punción del talón es un procedimiento doloroso y evaluado en la mayoría de los ensayos clínicos, incluida la aplicación de la escala Premature Infant Pain Profile (PIPP), para estudios con prematuros. Además, se encontró que la sacarosa oral al $24 \%$ fue la intervención más comparada con otras y que fue inferior o comparable al método canguro y la ingesta de leche materna para el control del dolor en los recién nacidos hospitalizados en la UCI. Conclusión: esta revisión señaló la efectividad clínica de intervenciones de diferente naturaleza evidenciada en los estudios rescatados. El olfato, el oído, el gusto y el tacto han sido campos potenciales de intervención para el control analgésico o la distracción del dolor en recién nacidos sometidos a procedimientos dolorosos de rutina. Es de destacar que el método canguro, la ingesta de leche materna y su olor, así como el sonido de los latidos del corazón materno han mostrado resultados positivos, seguros y reproducibles, incluso en comparación con el uso de sacarosa oral al $24 \%$. Es de destacar que nuevos ensayos clínicos y metaanálisis son fundamentales para fortalecer las conclusiones de este estudio.

Palabras clave: Unidades de Cuidados Intensivos; Neonatales; Manejo del dolor; Pediatría.

\section{Introdução}

Há décadas, acreditava-se que, por não conseguir formar memórias, os neonatos não seriam capazes de interpretar a dor. Hoje, sabe-se que naturalmente, bebês recebem, interpretam e respondem a estímulos nociceptivos ambientais e internos, de forma a sofrer com dor, com evidências que demonstram esse ser um processo organicamente semelhante e mais intenso em comparação ao dos adultos - com menor limiar e tolerância à dor -, com participação de neurônios nociceptores, corno dorsal da medula e tálamo. Em média, por volta da vigésima quarta semana de gestação há a fusão das fibras do corno dorsal da medula ao tálamo, o que possibilita a percepção do estímulo nociceptivo; já a mielinização acontece por volta da trigésima sétima semana. Quanto aos neonatos, sabe-se que a dor não tratada e repetitiva pode acarretar prejuízos a longo prazo a diversos sistemas complexos do corpo, inclusive ao cérebro. Hoje, há lacunas no conhecimento, avaliação e tratamento da dor que permitem a persistência de tais prejuízos em certo grau (Allegaert; Van Den Anker, 2016; Perry et al., 2018).

A avaliação e a quantificação da dor em neonatos são difíceis devido às suas condições cognitiva e física, porém, pesquisas têm demonstrado o desempenho sensível de escalas de dor aguda que auxiliam as equipes em saúde no controle analgésico dessa população. Há Neonatal Pain, Agitation, and Sedation Scale (N-PASS), a COMFORTneo e a Neonatal Infant Pain Scale (NIPS), que, inclusive, podem direcionar o tratamento por uma via não-farmacológica, a qual é potencial terapêutica, pois age diretamente ativando as vias inibitórias descendentes ou bloqueando a transmissão nociceptiva. O contato pele a pele, a flexão de tronco e uma única dose de substância doce, por exemplo, são medidas importantes em uma UTI neonatal que submete os pacientes a repetidos procedimentos invasivos e o manejo efetivo da dor em neonatos refere a qualidade do cuidado dos Serviços (Allegaert; Van Den Anker, 2016; Perry et al., 2018).

Frente a isso, ressalta-se a importância de se rever quais são as evidências mais atuais acerca do tratamento não farmacológico da dor em neonatos internados em UTI.

\section{Metodologia}

Trata-se de uma Revisão Integrativa de Literatura iniciada com busca de artigos nas bases de dados Literatura Latino Americana e do Caribe em Ciências da Saúde (LILACS), Scientific Electronic Library Online (SciELO) e Medical Literature Analysis and Retrieval Sistem (Medline) em setembro de 2021. Os descritores "Intensive Care Units, Neonatal" e "Pain management" juntos pelo operador booleano “AND” foram utilizados na busca em todas bases de dados. Para esta revisão, foram incluídas publicações de 2017 a 2021, sem restrições quanto ao idioma. Foram excluídos artigos revisão, metanálises e relatos de casos. Também, excluiu-se os artigos cujo título e/ou resumo não eram compatíveis com o propósito deste trabalho. 
Após isso, leu-se os 18 artigos resgatados após a aplicação dos critérios de exclusão por completo e organizou-se as informações de suas identificações, metodologias e resultados em uma adaptação de um instrumento de coleta de dados validado por Ursi (2005). Por fim, 14 artigos foram resgatados. O fluxograma abaixo demonstra o processo de exclusão dos artigos inicialmente distintos (Ursi, 2005).

Figura 1 - Fluxograma que explicita as etapas de exclusão de artigos inicialmente resgatados.

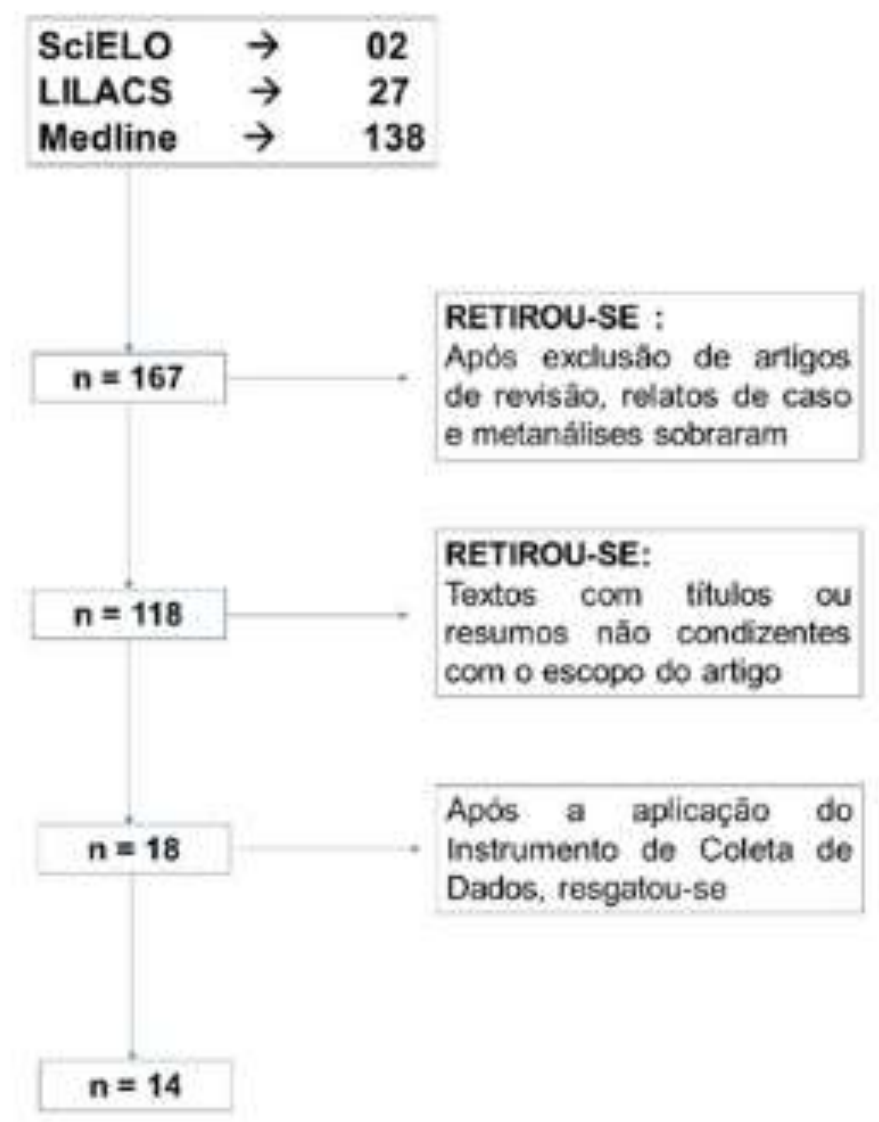

Fonte: Autores (2021).

\section{Resultados e Discussão}

Quadro 1 - Sistematização dos artigos resgatados.

\begin{tabular}{|c|l|l|}
\hline $\begin{array}{c}\text { AUTORES, } \\
\text { ANO }\end{array}$ & $\begin{array}{c}\text { DESENHO } \\
\text { DO ESTUDO }\end{array}$ & OBJETIVOS, RESULTADOS E CONCLUSÕES \\
\hline $\begin{array}{c}\text { CAMPBELL- } \\
\text { YEO et al., }\end{array}$ & $\begin{array}{c}\text { Ensaio Clínico } \\
\text { Randomizado }\end{array}$ & $\begin{array}{l}\text { Verificar a eficácia do uso do método canguru e da sacarose oral a 24\% isolados e combinados no } \\
\text { alívio da dor em 242 neonatos pré-termos internados em UTI neonatal participaram da randomização } \\
\text { e foram avaliados pela e Premature Infant Pain Profile (PIPP). } \\
\text { No grupo que recebeu o método canguru com o placebo houve a necessidade - por variados motivos } \\
\text { logísticos da pesquisa, como a não presença da mãe e PIPP > 6 - de uso de 1 dose de sacarose a 24\% } \\
\text { em } 22 \text { crianças. Os autores consideraram essa exposição como mínima e incapaz de alterar seus } \\
\text { resultados. Concluiu-se que o método canguru isolado tem importante eficácia em comparação com o } \\
\text { uso da sacarose a 24\%, podendo ser um importante método não farmacológico de controle da dor em } \\
\text { crianças pré-termo internadas em UTI neonatal que sejam submetidas a repetidos procedimentos } \\
\text { dolorosos. Também, destacam que a adição de sacarose a 24\% ao método canguru não apresentou } \\
\text { maiores benefícios em comparação com o método canguru isolado. Com isso, reforçam a a }\end{array}$ \\
\hline
\end{tabular}




\begin{tabular}{|c|c|c|}
\hline & & $\begin{array}{l}\text { necessidade de que se reveja o uso de sacarose a } 24 \% \text { como um método de primeira escolha para esse } \\
\text { objetivo. Não foram notadas complicações no desenvolvimento dos bebês relacionadas às } \\
\text { intervençôes. }\end{array}$ \\
\hline $\begin{array}{l}\text { STEVENS et } \\
\text { al., } 2018\end{array}$ & $\begin{array}{l}\text { Ensaio Clínico } \\
\text { Randomizado }\end{array}$ & $\begin{array}{l}\text { Trata-se de um estudo que verificou qual seria a dose mínima de sacarose a } 24 \% \text { para redução da dor } \\
\text { causada por punção de calcanhar em recém-nascidos internados em UTI neonatal. Dessa pesquisa, } \\
\text { participaram } 245 \text { neonatos internados em UTI neonatal randomizados em três grupos, um que } \\
\text { recebeu dose oral de } 0,1 \mathrm{ml} \text { de sacarose a } 24 \% \text {, outro com } 0,5 \mathrm{ml} \text { e outro com } 1,0 \mathrm{ml} \text {. Os pacientes } \\
\text { foram avaliados pelo Premature Infant Pain Profile-Revised (PIPP-R), os neonatos que receberam } \\
\text { diferentes doses de sacarose não apresentaram diferença significativa na avaliação da dor, de forma } \\
\text { que } 0,1 \mathrm{ml} \text { se mostrou a dose mínima necessária para controle da dor dos neonatos submetidos a } \\
\text { punção de calcanhar nesse estudo. Ainda ressaltou a necessidade de se investigar os impactos do uso } \\
\text { de sacarose no controle da dor em neonatos a longo prazo. }\end{array}$ \\
\hline $\begin{array}{l}\text { KAHRAMAN } \\
\text { et al., } 2020\end{array}$ & $\begin{array}{l}\text { Ensaio Clínico } \\
\text { Randomizado }\end{array}$ & $\begin{array}{l}\text { Trata-se de uma randomização que estudou a influência que sons intrauterinos, voz da mãe gravada e } \\
\text { MiniMuffs têm na distração da dor em neonatos internados em UTI neonatal submetidos a punção de } \\
\text { calcanhar. A amostra foi constituída de } 80 \text { neonatos em sua primeira semana de nascimento que } \\
\text { foram randomizados em igual quantidade para receberem tais intervenções. O grupo controle recebeu } \\
\text { o tratamento padrão da unidade. Usando-se a escala ConfortNeo, a NIPS e o tempo de choro, o } \\
\text { estudo observou que o tempo médio de choro do grupo que recebeu o som intrauterino foi de } 6.5 \mathrm{~s} \text {, } \\
\text { enquanto o do grupo controle foi de } 34.93 \mathrm{~s} \text {. O grupo de melhor desempenho também sentiu menos } \\
\text { dor e ficou mais confortável. O estímulo sonoro teve melhores resultados em comparação com a } \\
\text { diminuição dos ruídos do ambiente com os MiniMuffs. }\end{array}$ \\
\hline $\begin{array}{l}\text { SHUKI } \\
\text { al., } 20\end{array}$ & $\begin{array}{l}\text { Estudo } \\
\text { Multicêntrico e } \\
\text { Longitudinal } \\
\text { randomizado } \\
\text { controlado }\end{array}$ & $\begin{array}{l}\text { Nesta pesquisa, comparou-se o contato pele a pele com o uso de sacarose no controle da dor em } \\
\text { neonatos pré-termo submetidos a punção de calcanhar. A amostra foi composta por } 100 \text { neonatos } \\
\text { internados em UTI neonatal randomizados nos grupos-intervenção. Os neonatos foram avaliados com } \\
\text { a escala PIPP e os dois métodos não apresentaram diferença estatística importante para controle da } \\
\text { dor causada pelo procedimento. }\end{array}$ \\
\hline $\begin{array}{c}\text { SEN; } \\
\text { MANAV, } \\
2020\end{array}$ & $\begin{array}{c}\text { Ensaio Clínico } \\
\text { Randomizado } \\
\text { Prospectivo }\end{array}$ & $\begin{array}{l}\text { Trata-se de uma comparação entre o método canguru e o uso de sacarose oral para alívio da dor em } \\
\text { neonatos pré-termo internados em UTI neonatal submetidos a punção de calcanhar e avaliados com a } \\
\text { PIPP. Na amostragem final, houve } 64 \text { neonatos pré-termo randomizados em dois grupos, um para o } \\
\text { contato pele a pele e outro para o uso de sacarose oral diante do procedimento invasivo. Segundo os } \\
\text { resultados dessa pesquisa, ambos os métodos se mostraram eficazes em diminuir a dor dos neonatos. } \\
\text { Entretanto, ressalta-se que o método canguru apresentou-se com melhores resultados estatísticos e } \\
\text { potencialmente mais seguro, uma vez que são conhecidos os efeitos adversos do uso da sacarose } \\
\text { nesse contexto. }\end{array}$ \\
\hline $\begin{array}{c}\text { PENG et al., } \\
2017\end{array}$ & $\begin{array}{c}\text { Estudo } \\
\text { Randomizado } \\
\text { Prospectivo }\end{array}$ & $\begin{array}{l}\text { Esta pesquisa teve o objetivo de comparar em neonatos internos em UTI os métodos de ingerir leite } \\
\text { materno antes da punção de calcanhar e de mantê-los em uma posição flexionada em relação à linha } \\
\text { média, sem contenção da cabeça ou do corpo para avaliação do controle da dor por esses métodos, } \\
\text { gerada pelo procedimento. A amostra estudada foi composta por } 109 \text { neonatos pré-termo internados } \\
\text { em UTI e submetidos a punção de calcanhar e avaliados segundo a PIPP. O resultados sugeriram que } \\
\text { o uso de leite materno oral, em neonatos submetidos à punção de calcanhar, adicionado ou não ao } \\
\text { uso da posição flexionada e das mãos aquecidas, tem efeito analgésico. A mudança de posição se } \\
\text { mostrou mais eficaz na recuperação da dor do que na sua diminuição em si. Os bebês que receberam } \\
\text { a sucção, o leite materno e o afago da posição tiveram menos risco de ter dor leve a moderada } \\
\text { durante o procedimento em comparação aos bebês que receberam os cuidados de rotina da unidade. }\end{array}$ \\
\hline $\begin{array}{l}\text { TEKGUNDUZ } \\
\text { et al., } 2019\end{array}$ & $\begin{array}{c}\text { Estudo } \\
\text { Randomizado } \\
\text { Duplo-Cego } \\
\text { Controlado }\end{array}$ & $\begin{array}{l}\text { Esta pesquisa investigou se o uso de glicose oral e de canções de ninar causavam alívio da dor em } \\
\text { neonatos prematuros que eram submetidos a pressão nasal positiva em procedimentos como retirada } \\
\text { e reinserção do tubo traqueal e de aspiração oronasofaríngea. Avaliados pela escala NIPS e PIPP, } 106 \\
\text { neonatos pré-termo internados em UTI foram randomizados em três grupos, o controle, o que } \\
\text { recebeu a glicose oral e o que foi exposto à canção de ninar. Segundo os resultados desse estudo, os } \\
\text { neonatos que utilizaram da glicose tiveram menos dor após os procedimentos em comparação com } \\
\text { aqueles que ouviram canções de ninar. Os autores ressaltam a importância do uso de métodos } \\
\text { farmacológicos associados. }\end{array}$ \\
\hline $\begin{array}{l}\text { CIRIK; EFE, } \\
2020\end{array}$ & $\begin{array}{c}\text { Estudo } \\
\text { Randomizado } \\
\text { Controlado }\end{array}$ & $\begin{array}{l}\text { Esse estudo teve como objetivo comparar os efeitos do leite materno ordenhado, do enfaixamento, da } \\
\text { flexão facilitada, da combinação entre leite materno ordenhado e enfaixamento, e da soma leite } \\
\text { materno ordenhado e flexão facilitada, além dos métodos de cuidados padrão da unidade sobre a dor } \\
\text { do bebê prematuro antes, durante e após os procedimentos de inserção de sonda orogástrica. } \\
\text { Foram estudados os efeitos em } 187 \text { neonatos pré-termo randomizados nesses seis grupos. Segundo os } \\
\text { resultados dessa pesquisa, o enfaixamento, o leite materno ordenhado e a flexão facilitada são } \\
\text { eficazes no controle da dor provocada pela inserção da sonda orogástrica, sendo a soma do } \\
\text { enfaixamento e leite materno a combinação com melhor desempenho e segurança. }\end{array}$ \\
\hline $\begin{array}{l}\text { MC GINNIS et } \\
\quad \text { al., } 2016\end{array}$ & $\begin{array}{c}\text { Ensaio } \\
\text { Randomizado } \\
\text { Controlado } \\
\text { Prospectivo }\end{array}$ & $\begin{array}{l}\text { Trata-se de uma randomização que verificou a efetividade da vibração mecânica local no alívio da } \\
\text { dor causada pela punção do calcanhar de neonatos a termo. N-PASS foi o instrumento utilizado para } \\
\text { avaliar a dor nos } 56 \text { neonatos desse estudo. Eles foram randomizados em dois grupos, um controle } \\
\text { que recebeu sacarose oral a } 24 \% \text { e um que recebeu a vibração mecânica junto à sacarose oral a } 24 \% \text {. } \\
\text { Não foram encontrados efeitos adversos relacionados à adição da vibração a sacarose e concluiu-se } \\
\text { que a vibração potencializa o efeito analgésico da sacarose. }\end{array}$ \\
\hline
\end{tabular}




\begin{tabular}{|c|c|c|}
\hline $\begin{array}{l}\text { ALEMDAR; } \\
\text { TUFEKCI, } \\
2018\end{array}$ & $\begin{array}{l}\text { Estudo } \\
\text { Randomizado } \\
\text { Controlado }\end{array}$ & $\begin{array}{l}\text { Esse estudo objetivou verificar os efeitos na dor causada por aspiração por meio da exposição de } 92 \\
\text { neonatos pré-termo internados em UTI e submetidos à aspiração ao som dos batimentos cardíacos } \\
\text { maternos previamente gravados. A } \\
\text { dor foi avaliada pela PIPP e o conforto pela Premature Infant Comfort Scale (PICS). A amostra foi } \\
\text { randomizada em dois grupos, um que recebeu os sons cardíacos da mãe e outro que recebeu o } \\
\text { tratamento de rotina da UTI. Entre o grupo controle e intervenção houve significativa diferença } \\
\text { estatística que demonstrou ser o uso de sons cardíacos maternos gravados uma importante ferramenta } \\
\text { não-farmacológica de controle da dor causada por aspiração em neonatos pré-termo internos em UTI. }\end{array}$ \\
\hline $\begin{array}{l}\text { LAGO et al., } \\
2020\end{array}$ & $\begin{array}{l}\text { Estudo } \\
\text { Randomizado } \\
\text { Controlado } \\
\text { Duplo-cego }\end{array}$ & $\begin{array}{l}\text { Tal estudo demonstrou os efeitos analgésicos de duas doses repetidas de sacarose oral a } 24 \% \text { em } \\
\text { neonatos pré-termos internados em UTI e submetidos a punção de calcanhar. Foram eleitos } 72 \\
\text { neonatos pré-termo submetidos a punção de calcanhar, numa randomização em dois grupos; um } \\
\text { grupo que recebeu a dose repetida de sacarose e outro que recebeu uma dose padrão após punção de } \\
\text { calcanhar. Sua dor foi avaliada pela escala PIPP. Concluiu-se que repetir a dose de sacarose oral a } \\
24 \% \text { na população em estudo não se mostrou mais eficaz em comparação a uma única dose. Não } \\
\text { houve eventos adversos registrados no grupo intervenção. }\end{array}$ \\
\hline $\begin{array}{l}\text { COLLADOS- } \\
\text { GOMEZ et al., } \\
2017\end{array}$ & $\begin{array}{l}\text { Ensaio Clínico } \\
\text { Randomizado } \\
\text { Multicêntrico }\end{array}$ & $\begin{array}{l}\text { Ao comparar o uso de leite materno e sacarose a } 24 \% \text { para o controle da dor em neonatos pré-termo } \\
\text { que realização punção venosa, apontou-se que as duas intervenções têm eficácias comparáveis. } \\
\text { Participaram deste estudo } 63 \text { neonatos cuja dor causada pelo procedimento foi avaliada pela PIPP. }\end{array}$ \\
\hline $\begin{array}{l}\text { WIILLAMS et } \\
\text { al., } 2019\end{array}$ & $\begin{array}{l}\text { Estudo } \\
\text { Randomizado } \\
\text { Controlado }\end{array}$ & $\begin{array}{l}\text { Essa pesquisa objetivou examinar os efeitos do robô Calmer na variabilidade da frequência cardíaca } \\
\text { durante a punção venosa em neonatos pré-termo. O robô grava a menor frequência cardíaca e } \\
\text { respiratória maternas, que foram reproduzidas para o neonato antes da coleta de sangue, que também } \\
\text { receberam uma chupeta. Desse estudo, participaram } 14 \text { neonatos pré-termos internados em UTI seus } \\
\text { resultados sugerem que o Calmer tem potencial em melhorar os efeitos negativos a longo prazo } \\
\text { causados pelo aumento do estresse em bebês prematuros internados em UTI. }\end{array}$ \\
\hline $\begin{array}{l}\text { CHANVILLE } \\
\text { et al., } 2017\end{array}$ & $\begin{array}{l}\text { Estudo } \\
\text { Randomizado } \\
\text { Duplo-Cego } \\
\text { Prospectivo } \\
\end{array}$ & $\begin{array}{l}\text { Essa pesquisa objetivou verificar se o odor do leite materno em recém-nascidos pré-termo teria efeito } \\
\text { analgésico - e qual seria ele - sobre a punção venosa. Participaram } 33 \text { neonatos pré-termo que foram } \\
\text { randomizados em um grupo que recebeu o odor e o controle. O odor de leite materno se mostrou } \\
\text { eficiente no controle analgésico de pré-termos submetidos à punção venosa e avaliados pela PIPP. }\end{array}$ \\
\hline
\end{tabular}

Fonte: Autores (2021).

Para além das limitações intrínsecas à metodologia deste artigo, ressaltamos que apenas artigos da base de dados PUBMED foram considerados correspondentes ao objetivo deste trabalho. Ainda, destaca-se que cerca de $21 \%$ dos artigos foram do ano de 2017, havendo a mesma porcentagem para os anos de 2019 e 2018; para 2020, houveram $30 \%$ e $20167 \%$. Todos os artigos resgatados foram randomizações.

Um ensaio clínico Canadense com 242 neonatos pré-termos internados em UTI randomizados em receber o método canguru com o placebo, o método canguru com sacarose oral a $24 \%$ e apenas sacarose oral a $24 \%$ objetivou verificar a eficácia do uso do método canguru no controle da dor causada por procedimentos repetidos. Nesse estudo, verificou-se que é necessária uma revisão quanto ao método de primeira escolha para o controle não farmacológico da dor em neonatos internados em UTI, pois tal pesquisa resultou eficácia notável do método canguru em comparação com o uso da sacarose a $24 \%$, não havendo superioridade na junção dos dois métodos em comparação com o método canguru isolado na população estudada (CampbellYeo et al., 2019).

Corroborando a isso, um ensaio clínico prospectivo e randomizado que comparou o método canguru com o uso de sacarose oral para alívio da dor em 64 neonatos pré-termo internados em UTI neonatal submetidos a punção de calcanhar e avaliados com a PIPP concluiu que, apesar de haver equivalência na eficácia dos dois métodos, o método canguru foi estatisticamente superior à sacarose e potencialmente mais seguro, tendo em vista que é natural e com menos efeitos adversos em comparação com o outro (Sen \& Manav, 2020).

Outra randomização, dessa vez Indiana e com 100 neonatos pré-termo hospitalizados em UTI demonstrou que o contato pele a pele e o uso de sacarose têm eficácia semelhante para o manejo da dor causada pela punção de calcanhar (Shukla et al., 2018). Um ensaio randomizado com 187 neonatos pré-termo encontrou que o enfaixamento, o leite materno ordenhado e a flexão facilitada são eficazes no controle da dor provocada pela inserção da sonda orogástrica, sendo a soma do enfaixamento com o leite materno a intervenção com melhor desempenho (Apaydin Cirik \& Efe, 2020). 
O uso de sacarose associado a vibração mecânica também pode ser benéfico para o controle da dor em neonatos submetidos a punção de calcanhar, foi o que apontou um estudo randomizado controlado americano realizado com 56 neonatos a termo internados em UTI (McGinnis et al., 2016).

Já uma pesquisa multicêntrica duplo-cega realizada com 245 neonatos internados em UTI neonatal com idade gestacional de 24 a 42 semanas randomizados em os que receberam dose oral de $0,1 \mathrm{ml}$ de sacarose a $24 \%$, os que receberam $0,5 \mathrm{ml}$ e os de $1,0 \mathrm{ml}$, verificou que $0,1 \mathrm{ml}$ se mostrou a dose mínima necessária para controle da dor dos neonatos submetidos a punção de calcanhar, segundo a avaliação da dor pela Premature Infant Pain Profile-Revised (PIPP-R) (Stevens, Bonnie; Yamada, Janet; Campbell-Yeo et al., 2018).

Um ensaio clínico controlado demonstrou que o uso de duas doses padrão de sacarose oral a $24 \%$ em neonatos prétermos internados em UTI e submetidos a punção de calcanhar para controle da dor do procedimento não é superior em efeito analgésico ao uso de apenas uma dose, assim como também não apresentou efeitos adversos; esse estudo foi conduzido com 72 bebês que foram avaliados pela escala PIPP (Lago et al., 2020).

Uma randomização objetivou comparar o uso de leite materno e sacarose a $24 \%$ para o controle analgésico em neonatos pré-termo submetidos a punção venosa com 63 neonatos cuja dor do procedimento foi avaliada pela escala PIPP. O uso de leite materno comparado ao de sacarose para alívio da dor em neonatos pré-termo se mostrou tão eficaz quanto. Ainda, o leite materno se mostrou mais acessível e seguro (Collados-Gomez et al., 2017).

Para além do uso de sacarose, a sucção não nutritiva e a ingesta de leite materno tem se mostrado estratégias eficazes para a redução de dor em neonatos pré-termo; uma randomização apresentou que o uso de leite materno oral em neonatos submetidos à punção de calcanhar, adicionado ou não ao uso da posição flexionada e das mãos aquecidas, tem efeito analgésico. Além disso, a parcela dos 109 bebês que bebês que recebeu a sucção não nutritiva, o leite materno e o afago de posição flexionada sobre a linha média com mãos aquecidas pontuou menos risco de desenvolver dor leve a moderada quando comparada aos bebês que receberam os cuidados de rotina naquela UTI (Peng et al., 2018). O odor do leite materno também mostrou resultados positivos em relação ao controle da dor em neonatos pré-termo avaliados por PIPP submetidos a punção venosa (Chanville et al., 2017).

Como forma de distração da dor durante a punção de calcanhar, uma pesquisa comparou sons intrauterinos, voz da mãe gravada e MiniMuffs com o grupo controle que recebeu o tratamento padrão da Unidade. A partir disso, os 80 neonatos que participaram da amostra e foram avaliados pelo tempo de choro, pela escala ConfortNeo e pela Neonatal Infant Pain Scale (NIPS) colaboraram para a seguinte conclusão: o estímulo sonoro teve melhores resultados em comparação com a diminuição dos ruídos do ambiente e o grupo que recebeu os sons intrauterinos teve, em média, $6.5 \mathrm{~s}$ de choro, enquanto o grupo controle apresentou aproximadamente 35s de média (Kahraman et al., 2020).

Outra randomização realizada na Turquia com 106 neonatos pré-termo concluiu que a glicose administrada de forma oral é mais eficaz no controle da dor causada por pressão nasal positiva - por aspiração oronasofaríngea e reinserção e retirada de tubo traqueal - do que o estímulo auditivo provocado por canções de ninar (Tekgündüz et al., 2019). Em outra pesquisa, 92 neonatos pré-termo internados em UTI e submetidos à aspiração foram distribuídos em dois grupos, um que recebeu os sons cardíacos da mãe e outro que recebeu o tratamento padrão da UTI para a dor causada por esse procedimento. Nesse estudo, o uso de sons cardíacos maternos gravados se mostrou uma importante ferramenta não-farmacológica de controle da dor nessa população (Kü̧̈ük Alemdar \& Güdücü Tüfekci̇, 2018).

Um estudo controlado objetivou verificar o efeito analgésico do robô Calmer na variabilidade da frequência cardíaca durante a punção venosa em neonatos pré-termo. Os 14 neonatos pré-termos internados em UTI e os resultados desse estudo sugerem que o Calmer demonstra potencial em reduzir o efeitos a longo prazo do aumento do estresse em bebês prematuros internados em uma UTI (Williams et al., 2019). 


\section{Conclusão}

Diante do que foi exposto, conclui-se que a dor neonatal é um fenômeno que precisa ser diagnosticado e tratado para que se evitem prejuízos no desenvolvimento da criança. Ainda, ela pode ser avaliada por diversas escalas, como PIPP e NIPS e serem associadas ao tempo de choro e a scores que pontuem o conforto do neonato. Intervenções de diferentes naturezas se mostraram eficazes nos estudos resgatados; o uso de cheiros, sons, estímulo táctil e de paladar foram citados como potenciais analgésicos, dentre eles: método canguru, uso de sacarose oral a $24 \%$, odor de leite materno, sons de batimentos cardíacos maternos, ingesta de leite materno, flexão de tronco, aquecimento de mãos, vibração no membro que dói.

Também, houve superioridade em eficácia do método canguru e da ingesta do leite materno em comparação ao uso de sacarose oral a $24 \%$ em algumas das randomizações resgatadas. Frente a tal diversidade e importância do fortalecimento das evidências acerca do uso seguro e eficaz desses métodos, destaca-se que novos ensaios clínicos e metanálises são imprescindíveis para a disseminação de tais práticas e incorporação delas nos cuidados-padrão em UTIs neonatal.

\section{Referências}

Allegaert, K., \& Van Den Anker, J. N. (2016). Neonatal pain management: still in search of the Holy Grail. International Journal of Clinical Pharmacology and Therapeutics, 54(7), 514.

Apaydin Cirik, V., \& Efe, E. (2020). The effect of expressed breast milk, swaddling and facilitated tucking methods in reducing the pain caused by orogastric tube insertion in preterm infants: A randomized controlled trial. International Journal of Nursing Studies, 104 , 103532. https://doi.org/10.1016/j.ijnurstu.2020.103532

Collados-Gómez, L., Ferrera-Camacho, P., Fernandez-Serrano, E., Camacho-Vicente, V., Flores-Herrero, C., García-Pozo, A. M., \& Jiménez-García, R. (2018). Randomised crossover trial showed that using breast milk or sucrose provided the same analgesic effect in preterm infants of at least 28 weeks. Acta Paediatrica, 107(3), 436-441.

Campbell-Yeo, M., Johnston, C. C., Benoit, B., Disher, T., Caddell, K., Vincer, M., Walker, C. D., Latimer, M., Streiner, D. L., \& Inglis, D. (2019). Sustained efficacy of kangaroo care for repeated painful procedures over neonatal intensive care unit hospitalization: a single-blind randomized controlled trial. Pain, 160(11), 2580-2588. https://doi.org/10.1097/j.pain.0000000000001646

Chanville, A. B. De, Brevaut-malaty, V., Garbi, A., Tosello, B., \& Baumstarck, K. (2017). Analgesic Effect of Maternal Human Milk Odor on Premature Neonates : A Randomized Controlled Trial. https://doi.org/10.1177/0890334417693225

Kahraman, A., Gümüş, M., Akar, M., Sipahi, M., Bal Yılmaz, H., \& Başbakkal, Z. (2020). The effects of auditory interventions on pain and comfort in premature newborns in the neonatal intensive care unit; a randomised controlled trial. Intensive and Critical Care Nursing, 61(xxx). https://doi.org/10.1016/j.iccn.2020.102904

Küçük Alemdar, D., \& Güdücü Tüfekcİ, F. (2018). Effects of maternal heart sounds on pain and comfort during aspiration in preterm infants. Japan Journal of Nursing Science, 15(4), 330-339. https://doi.org/10.1111/jjns.12202

Lago, P., Cavicchiolo, M. E., Mion, T., Dal Cengio, V., Allegro, A., Daverio, M., \& Frigo, A. C. (2020). Repeating a dose of sucrose for heel prick procedure in preterms is not effective in reducing pain: a randomised controlled trial. European Journal of Pediatrics, 179(2), 293-301. https://doi.org/10.1007/s00431019-03509-2

McGinnis, K., Murray, E., Cherven, B., McCracken, C., \& Travers, C. (2016). Effect of vibration on pain response to Heel Lance. Advances in Neonatal Care, 16(6), 439-448. https://doi.org/10.1097/ANC.0000000000000315

Peng, H. F., Yin, T., Yang, L., Wang, C., Chang, Y. C., Jeng, M. J., \& Liaw, J. J. (2018). Non-nutritive sucking, oral breast milk, and facilitated tucking relieve preterm infant pain during heel-stick procedures: A prospective, randomized controlled trial. International Journal of Nursing Studies, 77, 162-170. https://doi.org/10.1016/j.ijnurstu.2017.10.001

Perry, M., Tan, Z., Chen, J., Weidig, T., Xu, W., \& Cong, X. S. (2018). Neonatal pain: perceptions and current practice. Critical Care Nursing Clinics, 30(4), 549-561.

Sen, E., \& Manav, G. (2020). Effect of Kangaroo Care and Oral Sucrose on Pain in Premature Infants: A Randomized Controlled Trial. Pain Management Nursing, 21(6), 556-564. https://doi.org/10.1016/j.pmn.2020.05.003

Shukla, V., Chapla, A., Uperiya, J., Nimbalkar, A., Phatak, A., \& Nimbalkar, S. (2018). Sucrose vs. skin to skin care for preterm neonatal pain control—a randomized control trial. Journal of Perinatology, 38(10), 1365-1369. https://doi.org/10.1038/s41372-018-0193-9

Stevens, Bonnie; Yamada, Janet; Campbell-Yeo, M. S. G., Harrison, Denise; Dionne, Kimberley; Taddio, Anna; McNair, Carol; Willan, A. M. B. K. W. S. S., \& Riahi, C. E. A. S. J. S. C. V. S. (2018). The Minimally Effective Dose of Sucrose for Procedural Pain Relief in Neonates: A Randomized Controlled Trial. MCN The American Journal of Maternal/Child Nursing, 43(5), 297. https://doi.org/10.1097/NMC.0000000000000457

Tekgündüz, K. Ş., Polat, S., Gürol, A., \& Apay, S. E. (2019). Oral Glucose and Listening to Lullaby to Decrease Pain in Preterm Infants Supported with NCPAP: A Randomized Controlled Trial. Pain Management Nursing, 20(1), 54-61. https://doi.org/10.1016/j.pmn.2018.04.008

Ursi, E. S. (2005). Prevenção de lesões de pele no perioperatório: revisão integrativa da literatura. Revista Latino-Americana de Enfermagem, $14(1)$, 2-127.

Williams, N., Maclean, K., Guan, L., Collet, J. P., \& Holsti, L. (2019). Pilot Testing a Robot for Reducing Pain in Hospitalized Preterm Infants. https://doi.org/10.1177/1539449218825436 